\title{
Treading a fine line: is diagnosing depression in young people just medicalising moodiness?
}

Depressed mood in young people is common, with a prevalence of up to $20 \%$ among teenagers consulting their GP. ${ }^{1}$ Depression during the teenage years is associated with functional impairment and health risk behaviours; ${ }^{2,3}$ episodes can be severe and prolonged, often not resolving over 18 months. ${ }^{4}$ Adolescent depression is a chronic and relapsing condition with a high level of continuity into adulthood, ${ }^{5,6}$ where it is associated with impaired psychosocial functioning. ${ }^{2}$ Even young people with low levels of depressive symptoms and associated impairment - so-called 'subsyndromal depression' - have been shown to have persistent impairment. ' There is some evidence that intervention can reduce psychological morbidity and it is therefore possible that early intervention could alter the experience of mental illhealth in later life. ${ }^{8}$ However, young people with psychological morbidity are difficult to engage in psychological therapies, ${ }^{9}$ even though such therapies appear to reduce symptoms and case prevalence. ${ }^{10}$ This reluctance to engage reduces the capacity of GPs and their practice counsellors to review depressed teenagers. The NICE guidelines on depression in children and young people $e^{11}$ argue for enhanced detection and risk profiling in community settings, but the precise means to achieve this remain unclear.

GPs may be seen as being well placed to undertake detection and risk profiling tasks, but there are a number of reasons why they might not readily adopt this role. Although GPs believe that depression is becoming increasingly common among teenagers ${ }^{12}$ and do identify and react to most of those with severe psychological morbidity, they also fail to identify the majority of those with depressive disorders. ${ }^{9}$ There is some evidence that GPs spend less time in consultations with young people; ${ }^{10}$ some practitioners feel that teenagers are hard to communicate with and worry about overmedicalising their lives. ${ }^{13}$ Even when GPs perceive that young people have psychological problems they do not always explore these and a specific management or follow-up plan is put into place in only a minority of cases. ${ }^{14} \mathrm{~A}$ number of beliefs about young people may impede clinical engagement.

First, some believe that teenagers avoid visiting their GP, but it seems that the majority of registered young people consult their GP each year, and consultation rates are relatively high, at two to three per year. Most attend with physical symptoms; behavioural or emotional complaints account for only $2 \%$ of presentations. ${ }^{15}$ Although there is some (dated) evidence that many young people believe that their GP is unsympathetic, or feel uncomfortable in consultations with their family doctor, more recent research suggests that teenagers are generally satisfied with the care that they receive in general practice. ${ }^{16}$ Similarly, the attitudes of teenagers may be less of a barrier to general practice care than their perceived status as a special, problematic group might suggest. ${ }^{17}$

Second, mood changes are seen as part of normal teenage development. Between $75 \%$ and $80 \%$ of young people do not experience distress of any depth or significance during their teens, ${ }^{18}$ and the belief that young people are miserable, hyperemotional, and at war with their parents and the world does not correspond with the epidemiology of psychological distress in the teenage population. ${ }^{19}$ Seeing mood changes in young people as normal is in one sense congruent with the low prevalence of psychological disturbance in this age group, and with the resilience and optimism of young people. However, the assumption that adolescence is inevitably a time of emotional flux and intense angst could lead to significant psychological distress being mis-categorised as normal. The reluctance to view low mood and associated symptoms as depression appears to reflect a risk assessment that gives greater weight to the hazards of labelling unhappiness as depression, than to the identification of depression and intervention to minimise current and future impairment. In other words, the boundary between normal and abnormal is being drawn too far into pathological territory. This normalisation of depression in teenagers may be a consequence of taking a psychosocial view of their problems. For example, GPs in socioeconomically deprived areas may see depression as a 'normal response to life events' in a context of disadvantage, and may be reluctant to respond to such patients because of their inability to modify the underlying structural and social factors. ${ }^{20} \mathrm{~A}$ similar process could apply with depressed teenagers who may be seen within the family unit rather than as individuals, particularly if their difficulties appear to arise within seemingly intractable family circumstances.

Third, there is a tendency to challenge the usefulness of a psychiatric diagnosis in teenagers. This appears to be different from GPs' responses to depression in adults, where organisational issues, referral options, therapies, and stigma influence clinical thinking, but not rejection of depression as a valid conceptual category. ${ }^{21}$ In our view this aversion to a diagnosis is related, at least in part, to a perceived lack of therapeutic options. Drug treatment is the last therapeutic option for mild and moderate depression in young people, used only when cognitive behaviourial therapy (CBT) has been implemented without response, and even then only initiated by specialists. Similarly, the absence of a usable psychological intervention may inhibit thinking and action, especially when structured psychological approaches to depression for adults have proved too rigid for the problems encountered in general practice. Family therapy, which may seem a useful response to depression in those young people who are prepared to involve their families, addresses the relationship 
problems or poor social functioning that act as maintaining factors for depression, rather than the depression itself. ${ }^{22}$

There are two challenges for GPs working with young people. The first, and easier, is to reframe the teenage years in more accurate terms, abandoning the stereotype of distress as being normal and unavoidable. The Adolescence Working Party of the Royal College of General Practitioners has done much to achieve this. ${ }^{16,17}$ The second, and perhaps harder, challenge is to develop effective psychological interventions with a group that does not engage for long. There are signs that this is possible. For example, an intervention using a single dose of CBTderived therapy significantly increased case identification among GPs in one practice, where it was implemented within routine consultations, albeit with considerable variation between doctors in the use of the intervention. ${ }^{23}$ Nonetheless, there is a significant amount of development work to be done before the evidence base about therapeutic interventions is robust enough for GPs to engage with confidence in the assessment and risk profiling encouraged by NICE.

\section{Steve lliffe,}

Professor of Primary Care, Department of Primary Care, University College London, London.

\section{Georgina Williams,}

FY2, Lonsdale Medical Centre, London.

\section{Victoria Fernandez,}

Specialist Registrar in Adolescent and Child Psychiatry, Imperial College London, London.

\section{Mar Vila,}

Koplowitz Training Fellow, Academic Unit of Adolescent and Child Psychiatry, Imperial College London, London.

\section{Tami Kramer,}

Senior Clinical Research Fellow, Academic Unit of Adolescent and Child Psychiatry, Imperial College London and Central and North West London NHS Foundation Trust.

\section{Julia Gledhill,}

Honorary Clinical Senior Lecturer, Imperial College London and Consultant Child and Adolescent Psychiatrist, Central and North West London NHS Foundation Trust.

\section{Lisa Miller,}

GP, Lonsdale Medical Centre, London.

\section{REFERENCES}

1. Yates P, Kramer T, Garralda E. Depressive symptoms amongst adolescent primary care attenders. Levels and associations. Soc Psychiatry Psychiatr Epidemiol 2004; 39: 588-594.

2. Rao U, Hammen C, Daley S. Continuity of depression during the transition to adulthood: a 5-year longitudinal study of young women. J Am Acad Child Adolesc Psychiatry 1999; 38(7): 908-915.

3. Castillo Mezzich A, Tarter RE, Giancola PR, et al. Substance use and risky sexual behaviour in female adolescents. Drug Alcohol Depend 1997; 44(2-3): 157-166.

4. Goodman R, Ford T, Meltzer H. Mental health problems of children in the community: 18 month follow up. BMJ 2002; 324: 1496-1497.

5. Colman I, Wadsworth MEJ, Croudace TJ, Jones PB. Forty-year psychiatric outcomes following assessment for internalizing disorder in adolescence. Am J Psychiatry 2007; 164(1): 126-133

6. Lewinsohn PM, Clarke GN, Seeley JR, Rhode P. Major depression in community adolescents: age at onset, episode duration, and time to recurrence. J Am Acad Child Adolesc Psychiatry 1994; 33(6): 809-818.

7. Angold A, Costello EJ, Farmer E, et al. Impaired but undiagnosed. J Am Acad Child Adolesc Psychiatry 1999; 38(2): 129-137.

8. Jacobson L, Churchill R, Donovan C, et al. Tackling teenage turmoil: primary care recognition and management of mental ill health during adolescence. Fam Pract 2002; 19(4): 401-409.

9. Westman A, Garralda ME. Mental health promotion for young adolescents in primary care: a feasibility study. $\mathrm{Br}$ J Gen Pract 1996; 46: 317.

10. Harrington R, Whittaker J, Shoebridge P, Campbell F. Systematic review of efficacy of cognitive behaviour therapies in childhood and adolescent depressive disorders. BMJ 1998; 316: 1559-1563.

11. National Institute for Clinical Excellence. Depression in children and young people: identification and management in primary, community and secondary care. Clinical guideline 28. http://www.nice.org.uk/guidance/CG28 (accessed 20 Jan 2009).

12. Vandana J, Ambelas A. A general practitioner perceptions and practice related to adolescent depressive presentations. Clin Child Psychol Psychiatry 2004; 9(3): 341-346.

13. Iliffe S, Gledhill J, da Cunha F, et al. The recognition of adolescent depression in general practice: issues in the acquisition of new skills. Primary Care Psychiatry 2004; 9: $51-56$

14. Martinez R, Reynolds S, Howe A. Factors that influence the detection of psychological problems in adolescents attending general practices. Br J Gen Pract 2006; 56: 594-599.

15. Kramer T, Iliffe S, Murray E, Waterman S. Which adolescents attend the GP? Br J Gen Pract 1997; 47 (418): 327.

16. Jacobson L, Mellanby A, Donnovan C, et al. Teenagers' views on general practice consultations and other medical advice. Fam Pract 2000; 17; 156-158.

17. Churchill R, Allen J, Denman S, et al. Do the attitudes and beliefs of young teenagers towards general practice influence actual consultation behaviour? Br J Gen Pract 2000; 50: 953-957.

18. Department of Health. The mental health of children and adolescents in Great Britain. London: HMSO, 2000.

19. Arnett JJ. Suffering, selfish, slackers? Myths and reality about emerging adults. J Youth Adolesc 2007; 36: 23-29.

20. Chew-Graham CA, Mullin S, May CR, et al. Managing depression in primary care: another example of the inverse care law? Fam Pract 2002; 19(6): 632-637.

21 Railton S, Mowatt H, Bain J. Optimizing the care of patients with depression in primary care: the views of general practitioners. Health Soc Care Community 2000; 8(2): 119-128.

22. Hodes M, Garralda E. NICE guidelines on depression in children and young people: not always following the evidence. Psychiatric Bulletin 2007; 31:361-362.

23. Gledhill J, Kramer T, Iliffe S, Garralda ME. Training general practitioners in the identification and management of adolescent depression within the consultation: a feasibility study. J Adolesc 2003; 245-250.

DOI: 10.3399/bjgp09X407081

\section{ADDRESS FOR CORRESPONDENCE}

\section{Steve Iliffe}

University College London, Department of

Primary Care, Hampstead Campus,

Rowland Hill Street, London, NW3 2PF.

E-mail: s.iliffe@pcps.ucl.ac.uk

\section{Cannabis and risk}

Although cannabis is less harmful to health than tobacco and alcohol and not in the same league as other drugs such as cocaine and heroin, regular use, over time, is associated with significant health risks. ${ }^{1}$ To date the discussions around cannabis have focused, rather unhelpfully, on whether or not cannabis should be legalised, or which Class (of the Misuse of Drugs Act, 1971) cannabis should be placed. At the end of January this year cannabis was reclassified by the government from a Class $\mathrm{C}$ to a Class $\mathrm{B}$ drug. This debate has served as a distraction to the real issue of how to help 